\title{
Expression and Prognostic Implications of FOXO3a and Ki67 in Lung Adenocarcinomas
}

\author{
Hong-Bin Liu ${ }^{1 \&}$, Xiang-Xiang Gao ${ }^{2 \&}$, Qing Zhang ${ }^{3}$, Jian $\mathrm{Liu}^{4}$, Yuan Cui ${ }^{3}$, Yan \\ $\mathrm{Zhu}^{1 *}$, Yi-Fei Liu ${ }^{3 *}$
}

\begin{abstract}
To investigate the significance of FOXO3a and Ki67 in human lung adenocarcinomas. Envision immunohistochemical staining and Western blotting were used to examine the protein expression of FOXO3a in 127 cases of human lung adenocarcinoma specimens. The positive rate in lung adenocarcinoma $(\mathbf{5 5 . 9 \%})$ was lower than that in normal tissues $(\mathbf{8 0 \%})$. We found that the expression of FOXO3a was closely related with the degree of differentiation, TNM staging, lymph node metastasis and survival. In addition, significant differences in the different pathological types of lung adenocarcinoma cases $(P<0.01)$. The FOXO3a positive rate of the acini as the main type (APA) $(86.7 \%)$ and the lepidic as the main type (LPA) $(82.4 \%)$ was higher than the solid as the main type (SPA) $(50.0 \%)$, the papilla as the main type (PPA) $(\mathbf{4 2 . 9 \%})$ and the micropapilla as the main type (MPA) (9.4\%). Moreover, the expression of FOXO3a was negatively related with Ki67 expression. Our results suggested that the expression of FOXO3a is closely correlated with the aggressiveness of lung adenocarcinoma. It was indicated that disregulation of FOXO3a might play key roles in the occurrence and development of lung adenocarcinoma and joint detection of the two markers might play an important role in diagnosing tumors.
\end{abstract}

Keywords: FOXO3a - Ki67 - lung adenocarcinoma - prognosis

Asian Pac J Cancer Prev, 16 (4), 1443-1448

\section{Introduction}

Lung cancer is becoming a common malignant tumor in recent years, along with smoking and environmental factors, the men with lung adnocarcinoma died of cancer is in the first place. Lung adnocarcinoma is one of the most important pathological types of lung cancer, but the histological type of lung adnocarcinoma has not formed a generally accepted diagnostic criteria. Recently, the International Association for the study of lung cancer (IASLC), America Thoracic Society (ATS) and the European Respiratory Society (ERS) published a new classification scheme of the 2011 International Multidisciplinary pulmonary adenocarcinoma (Travis et al., 2011). The "mixed adenocarcinoma" was further classified in the new classification scheme, which was more extrude the prominent effect of different pathological types in lung adenocarcinoma cancer, biological behavior, treatment and prognosis (Yoshizawa et al., 2011).

The FOX protein family was recently named the protein family, FOXO is the best entry subfamily in the FOX family, FOXO family is an important transcription factor, which is involved in cell metabolism, cell cycle/ apoptosis, and tumorigenesis etc.. FOXO3a is an important member of the family, its expression is reduced or absent in a variety of tumor types, which suggest that multiple mechanisms lead to inactivation of FOXO3a may promote tumor occurrence (Potente et al., 2005; Paik et al., 2007).

$\mathrm{Ki} 67$ is one of the most extensive cell proliferation marker at home and abroad in recent years.It can be expressed at all stages of the cell cycle exccept in G0 phase, because of its short half-life, it can be rapidly degraded after left the cell cycle, so it can be used to reflect the tumor proliferation rate. It has a high expression in many tumors and participate in tumors' occurrence, development, metastasis and prognosis (Wang et al., 2012). The relationship between the clinical outcome of Ki67 and lung cancer has been the focus of the teratment.

In this study we examined the effects of FOXO3a in the new classification of lung adenocarcinoma, and the correlation between FOXO3a and clinical pathological characteristics with Ki67, and explore the molecular pathogenesis of lung adenocarcinoma, then provide a new way for the treatment of the lung adenocarcinoma.

\section{Materials and Methods}

\section{Patients and tissue samples}

The sample is a collection of 127 surgically resected lung adenocarcinoma specimens with clear pathological 
diagnosis in Cardiothoracic Surgery, Affiliated Hospital of Nantong University during October 2005 to August 2009. All the cases are primary ones without radiotherapy, chemotherapy or other treatments before operation. Among all the cases, 61 are female patients and 66 are male ones. Their ages are from 39 to 80 , while the median age is 62 . All the required cases in the research were investigated by Affiliated Hospital of Nantong University Ethics Committee and were up to the standards in Declaration of Helsinki. All the cases are Hematoxylineosin staining paraffin tissue sections and were categorized by pathology attending physicians and above according to the new international multidisciplinary classification scheme standards of lung adenocarcinoma published by International Association for the Study of Lung Cancer (IASLC), American Thoracic Society (ATS) and European Respiratory Society (ERS). Among them, 17 are with lepidic as the main type (LPA), 45 are with acini as the main type (APA), 21 are with papilla as the main type (PPA), 32 are with micropapilla as the main type (MPA), 12 are with solid as the main type (SPA). All the tumor tissues were fixed by $10 \%$ neutral formalin for 24 to 48 hours, paraffin-embedded, sectioned serially (each slice is $4 \mu \mathrm{m}$ ) and FOXO3a, Ki67 immunohistochemically stained. Another 20 cases of normal lung tissues are introduced as a control group. Fresh specimens are placed in liquid nitrogen and stored at $-80{ }^{\circ} \mathrm{C}$.

\section{Follow-up}

Follow-up includes medical history and the patient's survival or not. Survival time is the interval between surgery date and the date of the follow-up on monthly basis.

\section{Reagent}

Imunohistochemical staining uses Envision method. The first antibodies are Rabbit Anti-Lambda Light Chain Monoclonal Antibody FOXO3a (American EPITOMICS Company) and Rabbit Anti-Lambda Light Chain Monoclonal Antibody Ki67 (American EPITOMICS Company) respectively. The second antibodies are all poly-HRP anti-mouse/rabbit IgG. They all use kits provided by Beijing ZhongShan Biotechnology Company, colored with DAB and counterstained by hematoxylin. Instead of the first antibodies, Phosphate Buffered Saline (PBS) is chosen as the negative contrast while FOXO3a and Ki67 are used as normal lung tissue specimens for positive contrast.

Western blotting adopts Electrophoretic Blot, with nitrocellulose membrane chosen as the adsorbing material. Tris-glycine buffer is chosen as the buffer, made up by $25 \mathrm{~mm}$ Tris, $192 \mathrm{~mm}$ glycine, PH8.3, containing 20\% (v/v) methanol. Blocking reagents are gelatin, Bovine Serum Albumin (BSA) and fat-free milk powder etc.

\section{Result determination}

FOXO3a and Ki67 are both located in Tumor cell nuclei, the positive cells are those with yellow to brown granules in the nucleus and whose staining intensity are higher than those with specific staining in the background. Under the optical microscope, observe 5 instances at high magnification $(\times 400)$ in each case randomly (count 100 tumor cells per high magnification). Staining intensity was scored as "0" (no staining), "1" (weakly stained), "2" (moderately stained), or " 3 " (strongly stained). Counting the number of the positive cells under objective lens as well, 0 score for negative, 1 score for the condition in which the percentage of stained cells is smaller than or equal to $10 \%$ within a field of vision, 2 scores for $11 \%$ $50 \%, 3$ scores for $51 \%-75 \%$ and 4 scores for the condition in which the percentage of stained cells is bigger than $75 \%$. At last, multiply these two scores: 0-3 scores is negative, expressed with "-", 3 scores is "+", 4 scores is “++", 5 scores and above are positive, expressed by " +++ " and "+ $+++"$ respectively.

\section{Statistical processing}

Statistical analysis was performed using the PASW statistics 20 software package. The relation between FOXO3a and Ki67 and clinicalpathological features was examined by $\chi^{2}$ testing. The correlation between FOXO3a and Ki67 are analyzed according to Spearman Rank Correlation. Survival analysis adopts KaplanMeier method. Differences were considered significant at $p<0.05$.

\section{Results}

The Expressions of FOXO3a and Ki67 in Lung Adenocarcinoma and Normal Lung Tissues

We detected the expression of FOXO3a and Ki67 by IHC in 127 cases of lung adenocarcinoma, and we found that FOXO3a protein positive ones are 71 (55.9\%), while the number is $16(80 \%)$ in normal lung tissues. The difference between them has statistical significance $(p<0.01)$. In 127 cases of lung adenocarcinoma, there are 89 cases $(70.1 \%)$ whose proliferation index of Ki67 is positive, apparently higher than the adjacent normal control group (5\%), indicating the difference between them also has statistical significance $(p<0.01)$.

The expression of FOXO3a was detected in lung adenocarcinoma and normal lung tissues by Western blot. There are differences between four representative paired samples of lung adenocarcinoma tissues and normal lung tissues. The stronger expression of FOXO3a in normal tissues than in the lung adenocarcinomas.

Relation between positive expression of $\mathrm{FOXO}$ a and clinical pathology in lung cancer tissues

The expression intensity of FOXO3a is related to histological differentiating degree of lung adenocarcinoma, new pathological classification, TNM staging, lymph node metastasis and lifetime. The respective positive rates of well, moderately and poorly differentiated groups are: well differentiated group (78.6\%), moderately differentiated group $(55.0 \%)$, poorly differentiated group (26.3\%) $(p<0.01)$. TNM staging: stage I $(81.0 \%)$, stage II $(46.7 \%)$, stage III $(29.4 \%)$, stage IV $(0 \%)(p<0.01)$. Positive group of lymph node metastasis $(33.9 \%)$ is lower than negative group (76.9\%) $(p<0.01)$. Median of lifetime is 44 months. The positive rate of " $<44$ months" $(28.6 \%)$ is lower than " $\geq 44$ months" (82.8\%). 
Relation of expressions of $\mathrm{FOXO} 3 \mathrm{a}$ and the new pathological classification of lung adenocarcinoma

According to the new pathological classification of lung adenocarcinoma, the expressions of FOXO3a have significant differences. The positive rates of cases with lepidic as the main type $(82.4 \%)$ and those with acini as the main type $(86.7 \%)$ are highest, followed by those with solid as the main type (50.0\%) and those with papilla as the main type $(42.9 \%)$, while the lowest is those with micropapilla as the main type $(9.4 \%),(p<0.01)$.

Correlation of the expressions in lung adenocarcinoma between FOXO3a and Ki67

All the cases in this experiment are FOXO3a and Ki67 stained simultaneously. There are 39 cases in which both FOXO3a and Ki67 showing positive expression, 32 cases in which FOXO3a showing positive expression while

Table 1. FOXO3a and Ki67 Expression and Clinicopathologic Parameters in 127 Lung Adenocarcinoma Specimens

\begin{tabular}{|c|c|c|c|c|c|}
\hline \multirow[t]{2}{*}{ Parameters } & \multirow[t]{2}{*}{ Total } & \multicolumn{2}{|c|}{ FOXO3a } & \multirow[t]{2}{*}{$\chi^{2}$} & \multirow[t]{2}{*}{$p$ value } \\
\hline & & high & low & & \\
\hline \multicolumn{6}{|l|}{ Gender } \\
\hline Male & 66 & 37 & 29 & 0.001 & 0.971 \\
\hline Female & 61 & 33 & 28 & & \\
\hline \multicolumn{6}{|l|}{ Age(year) } \\
\hline$<60$ & 48 & 22 & 26 & 3.176 & 0.075 \\
\hline$\geq 60$ & 79 & 49 & 30 & & \\
\hline \multicolumn{6}{|c|}{ Tumor size(cm) } \\
\hline$<3$ & 49 & 30 & 19 & 0.916 & 0.339 \\
\hline$\geq 3$ & 78 & 41 & 37 & & \\
\hline \multicolumn{6}{|c|}{ Lymph node metastasis } \\
\hline No & 65 & 50 & 15 & 23.859 & $<0.01$ \\
\hline Yes & 62 & 21 & 41 & & \\
\hline \multicolumn{6}{|l|}{ Stage } \\
\hline Well & 28 & 22 & 6 & 12.610 & 0.002 \\
\hline Moderately & 80 & 44 & 36 & & \\
\hline Poorly & 19 & 5 & 14 & & \\
\hline \multicolumn{6}{|l|}{ TNM } \\
\hline I & 58 & 47 & 11 & 31.916 & $<0.01$ \\
\hline II & 30 & 14 & 16 & & \\
\hline III & 34 & 10 & 24 & & \\
\hline IV & 5 & 0 & 5 & & \\
\hline \multicolumn{6}{|c|}{ Median survival(Month) } \\
\hline$<44$ & 63 & 18 & 45 & 37.891 & $<0.01$ \\
\hline$\geq 44$ & 64 & 53 & 11 & & \\
\hline \multicolumn{6}{|c|}{ Ki67 expression } \\
\hline High & 89 & 39 & 50 & 17.623 & $<0.01$ \\
\hline Low & 38 & 32 & 6 & & \\
\hline
\end{tabular}

Statistical analyses were performed by the Pearson $\chi^{2}$ test, $\mathrm{P}<0.05$ was considered significant

Table 2. The Relationship between FOXO3a Expression and New Pathological Classification of Lung Adenocarcinoma

\begin{tabular}{lrrrrr}
\hline \multirow{2}{*}{$\begin{array}{l}\text { New pathological } \\
\text { classification }\end{array}$} & Negative & \multicolumn{3}{c}{ Positive } & $\begin{array}{c}\text { Positive } \\
\text { rate }(\%)\end{array}$ \\
\cline { 3 - 4 } LPA & 3 & 1 & 6 & 7 & 82.4 \\
APA & 6 & 8 & 16 & 15 & 86.7 \\
SPA & 6 & 2 & 2 & 2 & 50 \\
PPA & 12 & 7 & 2 & 0 & 42.9 \\
MPA & 29 & 1 & 2 & 0 & 9.4 \\
\hline
\end{tabular}

Ki67 showing negative expression, 6 cases in which both FOXO3a and Ki67 showing negative expression, 50 cases in which FOXO3a showing negative expression while Ki67 showing positive expression. According to Spearman Correlation Analysis, the expressions of FOXO3a and $\mathrm{Ki} 67$ in 127 cases of lung adenocarcinoma tissues demonstrate a negative correlation. $(\mathrm{r}=-0.90, p<0.01)$.

Analysis on expression of FOXO3a and prognosis of the patients

We divide the patients according to gene expression profiles to positive group and negative group. (As shown in Figure 4) The median of survival time of FOXO3a positive group is 72 months, while that of the negative group is 16.5 months, indicating statistical difference in survival time between the two groups $(p<0.01)$.

The median of survival time of the 127 patients is 44 months. Using Kaplan-Meier analytical methods for survival statistics analysis, the results show that the median of the survival rate of FOXO3a positive group

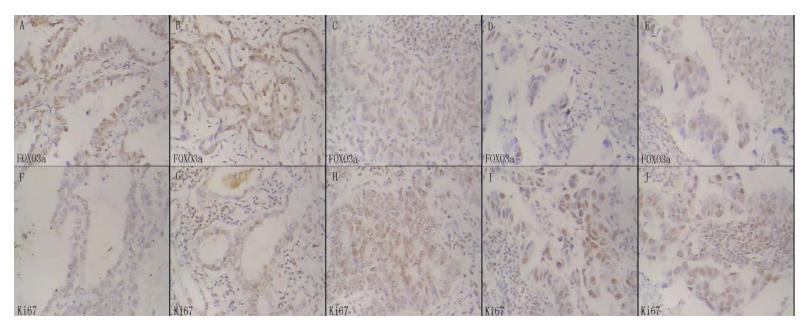

Figure 1. Expression of FOXO3a in the New Pathological Classification of Lung Adenocarcinoma Tissues(A:LPA, B:APA, C:SPA, D:PPA, E:MPA). The expression of Ki67 in the new pathological classification of lung adenocarcinoma tissues(F:LPA, G:APA, H:SPA, I:PPA, J:MPA). (Envision $\times 200$ )

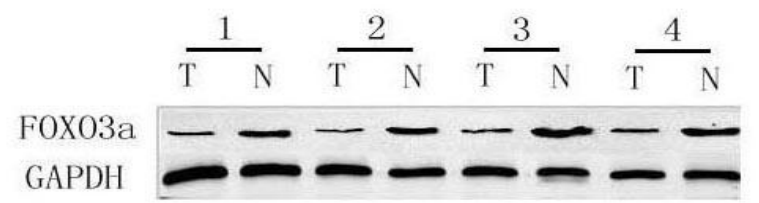

Figure 2. The Expression of FOXO3a was Detected in Lung Adenocarcinoma and Normal Lung Tissues. Expression of FOXO3a in four representative paired samples of lung adenocarcinoma tissue (T) and normal lung tissues $(\mathrm{N})$. GAPDH was used as a loading control. The same experiment was repeated at least three times

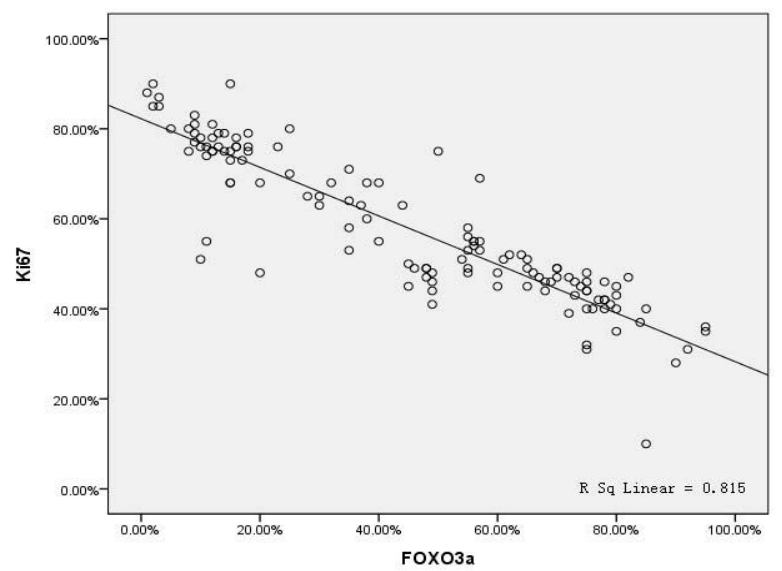

Figure 3. The Correlation of FOXO3a and Ki67 


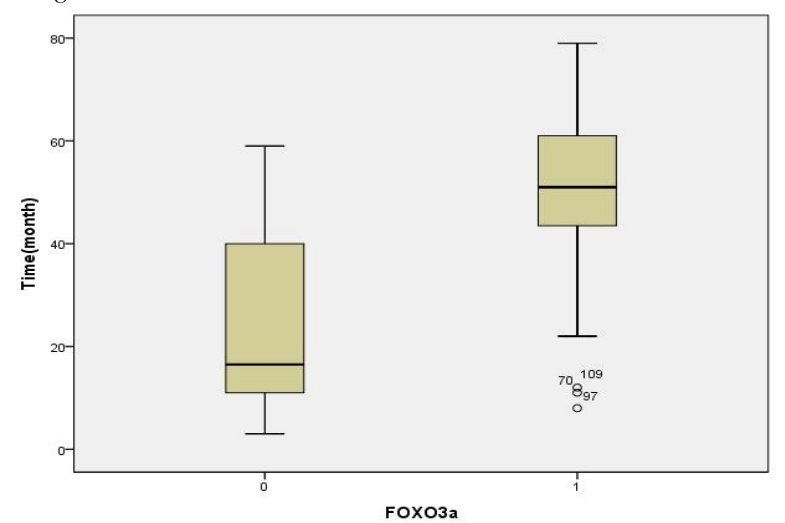

Figure 4. The Relevance of Research of FOXO3a and the Survival Time

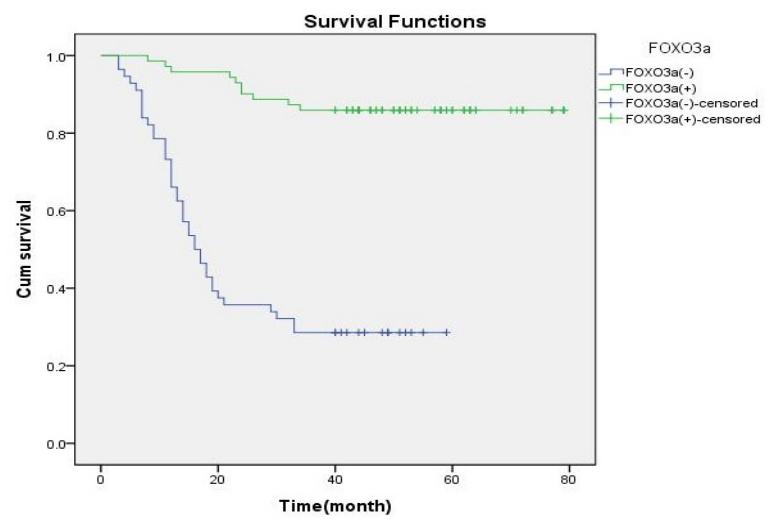

Figure 5. Overall Survival Curves of Lung Adenocarcinomna Positive/Negative of FOXO3a

is $82.8 \%$, while that of the negative group is $28.6 \%$, the differences of which are significant ( $p<0.01$, Figure 5).

\section{Discussion}

Lung cancer is one of the malignant tumors that severely threat people's health and life. It has high malignant degree and high death rate, the five-year survival rate of being just 10\%-15\%. In recent years, its morbidity and death rate are on a significant rise. The occurrence and development of lung cancer is a disease process involving multi-genes. As it hasn't been clear what the occurrence mechanism is so far, the treatment for lung cancer still lacks specificity. The biological characteristics of lung cancer are complicated, but as research continues, people have come to realize that different types of tumors have different driven genes and protein expressions (Drobniene et al., 2011) . FOX protein family is a protein family that got named recently, while FOXO is a subfamily, who is researched most profoundly, of FOX protein family. FOXO's activity level, sub-cellular localization and signaling pathway that it takes part in all closely related to the occurrence and development of tumors because FOXO is the transcription factor. Every member of the FOXO family includes a highly conserved DNA-binding domain in evolution, namely forkhead domain (Tsai et al., 2007; Ge et al., 2013). Forkhead box O3a, FOXO3a is one member of the FOXO family. The first time that people found the FOXO family is when their participation in chromosome translocation was found (Castrillon et al., 2003). FOXO3a is widely distributed in human body, such as stomach and intestine, liver, lung, breast, ovary, prostate, brain, peripheral blood leukocyte etc. Its activity is adjusted on multiple levels, including gene expression level, post-translational modifications, protein stability and protein-protein interaction. Among them, the main manifestation is its regulations on phosphorylation, acetylization, localization and degradation of sub-cells (Vogt et al., 2005) .

FOXO3a transcription factor is an important signal molecule in the signaling pathway of Insulin/ Phosphoinositide-3 Kinase (PI3K)/Akt (insulin-PI3K$\mathrm{Akt}$ ). This pathway has some negative regulatory impact on the expression of FOXO3a (Castrillon et al., 2003; Tsai et al., 2007)-commonly finding PI3K-Akt signaling pathway be activated in tumors, leading to the imbalance of the expression of FOXO3a, manifesting as low or no expression (Ge et al., 2013). Induced by the insulin-like growth factor, PI3K/Akt pathway is activated. Akt makes 3 key phosphorylation sites of FOXO3a (Thr32, Ser253, Ser315) phosphorylate. The phosphorylated FOXO3a meets falling affinity with DNA, but increasing affinity with 14-3-3 protein. It then transports from the nucleus to the cytoplasm. Brunet and some other scholars believe that 14-3-3 protein binds with FOXO3a protein in the nucleus (Brunet et al., 2002). By inducing the changes on the conformation of FOXO3a molecular, nuclear export sequence is exposed and the protein can also interact with the transporters outside the nuclear, thus promoting FOXO3a protein transporting from the nucleus to the cytoplasm. 14-3-3 protein binds with FOXO3a protein in the cytoplasm, making FOXO3a protein retaining in it so as to inhibit FOXO3a's activity (SO et al., 2003; Huang et al., 2005; Dansen et al., 2009; Levanon et al., 2014). Research results of Habashy and others show that FOXO3a that located in the cell nucleus can mediate the expression of cell cycle inhibitors like CDK etc, however, the pathway of PI3K/AKt obstructs the expression of FOXO3a in the cell nucleus by phosphorylating it, making a poor prognosis. Apart from Akt, there are also some other kinases which can phosphorylate FOXO protein (Habashy et al., 2011). Now, it has been found that the phosphorylation of FOXO3a and the output of the nucleus exist also in lung cancer, liver cancer, breast cancer, breast cancer and glioblastoma. Therefore, considering FOXO3a's important role in apoptosis and cancer development, inactivation of FOXO3a caused by different mechanisms may promote the occurrence and development of cancer (Nho et al., 2014).

At present, preliminary studies have been done on expressions of FOXO3a in various tumor tissues both at home and abroad. Abnormal expression of FOXO3a has a close relationship with breast neoplasms. By injecting over-expressed estrogen-dependent breast cancer cell line MCF-7 cell line, as well as using technologies of immunohistochemistry and immunoblot, Zou and his colleges discover that in the estrogen receptor positive MCF-7 cells, expression of FOXO3a lowers regulator genes of ER, who play an important role in cell proliferation. They also discover that $\mathrm{FOXO} 3 \mathrm{a}$ raises cyclin-dependent kinase inhibitors-p21Cip1, p27Kip1 
and p57Kip2, inhibiting the proliferation of cancer cells. On the contrary, the knocking out of FOXO3a gene accelerates the growth of tumor cells (Zou et al., 2008). Chiacchiera and some other scholars found that a prolonged inactivity of P38a in rectal cancer can induce FOXO3a's translocation into cell nuclei, causing cell autophagy, thus leading to cell cycle arrest and cell death. By detecting the expression of FOXO3a protein in different histological types of breast cancer through immunohistochemical method (Chiacchiera et al., 2009), Jin and some other scholars discovered that FOXO3a was expressed in all the benign breast tumors and most of the FOXO3a (91\%-94\%) were widely present in the cytoplasm, being inactive. However, for the invasive ductal carcinoma, especially the two subtypes-scirrhus and intraductal papillary carcinoma, $83 \%$ of the cases expressed FOXO3a and $71 \%$ of them existed in the cell nuclei in activated form (Jin et al., 2004). Recent researches of Mudan Lu and his colleges proved that low expression of FOXO3a correlated significantly with poor prognosis of ovarian cancer (Lu et al., 2012). They divided 46 cases of clinicopathologically diagnosed ovarian cancer samples into FOXO3a high expression group and FOXO3a low expression group; and analyzed the relation between expression level and prognosis. It turned out that the expression of FOXO3a was related to tumor staging and lymph node metastasis, having little to do with prognostic factors like tumor size etc. The death rate of high expression group (29.63\%) had significant difference from that of the low expression group (57.89\%). Recently, they also found that FOXO3a phosphorylated patients had poor prognosis ( $\mathrm{Lu}$ et al., 2012). This might result from phosphorylated FOXO3a's location in cytoplasm, thus losing the activity. It can thus be observed that FOXO3a molecule is a significant kind of transcription factors, who plays an important role in apoptosis and occurrence of tumors. All these indicate that expression of FOXO3a has a close relationship with occurrence, development and metastasis of tumors. A disorder of its expression may promote the metastasis of lymph nodes. As regard to the relation between FOXO3a and the occurrence and development of lung adenocarcinoma, researches are still at the initial stage of development at the moment. In the lung adenocarcinoma caused by smoking afflicted mouse models, it was detected that $50.7 \%(38 / 75)$ of the mice were in a lack of FOXO3a homozygotes, while the percentage was just $10 \%(1 / 10)$ in the control group. This indicates that for the lung adenocarcinoma caused by smoking afflicted mice, absence of FOXO3a is of great significance. This result fell in line with that of Blake Jr.'s (Blake et al., 2010).

Previous studies have proven that both FOXO3a and Ki67 are connected with biological behaviors such as the growth and metastasis of tumors, affecting the development of tumors and patients' prognosis together (Ge et al., 2013). However, no study has yet very clearly elucidated what's the mechanism in which FOXO3a and Ki67 interact to lead to the malignant progression of tumors. Reports about the interaction between FOXO3a and Ki67 in human lung adenocarcinoma are very limited at home and abroad. This research has detected the expression of FOXO3a in 127 cases of lung adenocarcinoma specimens, finding that FOXO3a distributes mainly in cell nucleus and its positive rate in malignant tumor is significantly less than normal tissues. All these are consistent with previous reports regarding its performance in other tissue tumors (Fernández et al., 2008; Karger et al., 2009; Lu et al., 2009; Chen et al., 2010), indicating that the deregulation or reduction of the expression of FOXO3a protein may accelerate the occurrence and growth of lung adenocarcinoma. According to the analysis on the clinicopathological data of lung adenocarcinoma afflicted patients, the expression of FOXO3a is significantly related to the new pathological classification of lung adenocarcinoma. The positive rates of those with lepidic as the main type and those with acini as the main type are highest, also enjoying good prognosis. Next are those with solid as the main type and those with papilla as the main type. The lowest is those with micropapilla as the main type, prognosis also being poor $(p<0.01)$. These findings accord with reports of Yoshizawa and some other scholars that different types of invasive adenocarcinoma have significant differences in prognosis. Those with lepidic as the main type and those with acini as the main type enjoy rather good prognosis, while invasive mucous adenocarcinoma, colloid carcinoma and those with solid as the main type and those with papilla as the main type have rather poor prognosis (Russell et al., 2011; Yoshizawa et al., 2011). Apart from that, the positive rate of FOXO3a protein is significantly correlated to the level of malignancy of tumors, TNM staging and lymph node metastasis, while does not related to age, gender and the size of tumor. The expression of FOXO3a protein is related to the level of malignancy of lung adenocarcinoma, metastasis and invasive ability. The survival analysis shows that the median of survival time of patients in the FOXO3a protein positive expression group is higher than that of the negative expression group. The above results suggest that detecting the expression of FOXO3a protein clinically is favorable to judging the level of malignancy of lung adenocarcinoma and the prognosis of patient. Therapeutically, regulating the expression and distribution of FOXO3a protein in cancer tissues so as to promoting of the apoptosis of tumor cells can provide a new way for the treatment of cancer.

\section{References}

Blake Jr, Mikse OR, Freeman WM, et al (2010). FOXO3a elicits a pro-apoptotic transcription program and cellular response to human lung carcinogen nicotine-derived nitrosaminoketone (NNK). Lung Cancer, 67, 37-47.

Brunet A, Kanai F, Stehn J, et al (2002). 14-3-3 trans its to the nucleus and participates in dynamic nucleocytoplasmic transport.J Cell Biol, 156, 817-28.

Castrillon DH, Miao I, Kollipara R, et al (2003). Suppression of ovarian folic leactivation in mice by the transcription fator FOXO3a. Science, 301, 215-8.

Chen J, Gomes AR, Monteiro LJ, et al (2010). Constitutively nuclear FOXO3a localization predicts poor survival and promotes akt phosphorylation in breast cancer. PLoS One, 5, 12293.

Chiacchiera F, Simone C (2009). Inhibition of p38alpha unveils 
Hong-Bin Liu et al an AMPK-FoxO3A axis linking autophagy to cancer-specific metabolism. Autophagy, 5, 1030-3.

Dansen TB, Smits LM, Vantriest MH, et al (2009). Redox sensitive cysteines bridge p300/CBP-mediated acetylation and FOXO4 activity. Nat Chem Biol, 5, 664-72.

Drobniene M, Cic6niene A, Zelviene TP, et al (2011). Targeted therapy in patients with non-small cell lung cancer previously treated with chemotherapy. Medicina, 47, 520-5.

Fernández de Mattos S, Villalonga P, Clardy J et al (2008). FOXO3a mediates the cytotoxic effects of cisplatin in colon cancer cells. Mol Cancer Ther, 7, 3237-46.

Ge Y F, Sun J, Jin C J, et al (2013). AntagomiR-27a targets FOXO3a in glioblastoma and suppresses U87 cell growth in vitro and in vivo. Asian Pac J Cancer Prev, 14, 963-8.

Habashy HO, Rakha EA, Aleskandarany M, et al (2011). FOXO3a nuclear localization is associated with good prognosis in luminal-like breast cancer. Breast Cancer Res Treat, 129, 11-21.

Huang H, Regan KM, Wang F, et al (2005). Skp2 inhibits FOXO1 in tumor suppression through ubiquit in mediated degradation. Proc Natl Acad Sci USA, 102, 1649-54.

Jin GS, Kondo E, Miyake T, et al (2004). Expression and intracellular localization of FKHRL1 in mammary gland neoplasms. Acta Med Okayama, 58, 197-205.

Karger S, Weidinger C, Krause K, et al (2009). FOXO3a:a novel player in thyroid carcinogenesis? Endocr Relat Cancer, 16, 189-99.

Levanon K, Sapoznik S, Bahar-Shany K, et al (2014). FOXO3a loss is a frequent early event in high-grade pelvic serous carcinogenesis. Oncogene, 33, 4424-32.

Lu M, Ma J, Xue W, et al (2009). The expression and prognosis of FOXO3a and Skp2 in human hepatocellular carcinoma. Pathol OncolRes, 15, 679-87.

Lu M, Xiang J, Xu F, et al (2012). The expression and significance of pThr32-FOXO3a in human ovarian cancer. Medical Oncology, 29, 1258-64.

Lu M, Zhao Y, Xu F, et al (2012). The expression and prognosis of FOXO3a and Skp2 in human ovarian cancer. Medical Oncology, 29, 3409-15.

Nho R S, Hergert P (2014). FoxO3a and disease progression. World J Biol Chem, 5, 346-54.

Paik J H, Kollipara R, Chu G, et al (2007). FOXOs are lineagerestricted redundant tumor suppressors and regulate endothelial cell homeostasis. Cell, 128, 309-323.

Potente M, Urbich C, Sasaki K, et al (2005). Involvement of FOXO Transcription factors in angiogenesis and postnatal neovascularization. Clin Invest, 115, 2382-92.

Russell P A, Wainer Z, Wright G M, et al (2011). Does lung adenocarcinoma subtype predict patient survival? A clinicopathologic study based on the new international association for the study of lung cancer/American thoracic society/European respiratory society international multidisciplinary lung adenocarcinoma classification. $J$ Thorac Oncol, 2, 1-9.

SO CW, Cleary ML (2003). Common mechanism for oncogenic activation of MLL by forkhead family proteins. Blood, 101, 633-9.

Travis W D, Brambilla E, Noguchi M, et al (2011). International association for the study of lung cancer/american thoracic society/european respiratory society international multidisciplinary classification of lung adenocarcinoma. $J$ Thorac Oncol, 6, 244-85.

Tsai KL, Sun YJ, Huang CY, et al (2007). Crystal structure of the human FOXO3a-DBD/DNA complex suggests the effects of post-translational modification. Nucleic Acids Res, 35, 6984-94.

Vogt PK, Jiang H, Aoki M (2005). Triple layer control:phosphorylation, acetylation and ubiquitination of FOXO proteins. Cell Cycle, 4, 908-13.

Wang J X, Zhang Y Y, Yu X M, et al (2012). Role of centromere protein $\mathrm{H}$ and Ki67 in relapse-free survival of patients after primary surgery for hypopharyngeal cancer. Asian Pac J Cancer Prev, 13, 821-5.

Yoshizawa A, Motoi N, Riely GJ, et al (2011). Impact of proposed IASLC/ATS/ERS classification of lung adenocarcinoma: prognostic subgroups and implications for further revision of staging based on analysis of 514 stage I cases. Mod Pathol, 24, 653-64.

Zou Y, Tsai WB, Cheng CJ, et al (2008). Forkhead box transcription factor FOXO3 a suppresses estrogen-dependent breast cancer cell proliferation and tumorigenesis. Breast Cancer Res, 10, 21. 